Discussion/conclusion Online surveys are an effective method of establishing important brand values for a sexual health service. Overall, respondents preferred a distinct identity for the service, exhibited through uniforms and a transparent naming convention. Though traditional barriers to accessing services persist, so also do the core values of confidentiality and professionalism.

\begin{tabular}{|c|c|c|c|c|c|c|c|c|}
\hline \multirow[t]{2}{*}{ Naming convention } & \multirow[t]{2}{*}{ Total } & \multicolumn{3}{|l|}{ Age } & \multicolumn{2}{|l|}{ Gender } & \multicolumn{2}{|c|}{$\begin{array}{l}\text { Service } \\
\text { user? }\end{array}$} \\
\hline & & $<25$ & $\begin{array}{l}25- \\
44\end{array}$ & $45+$ & Female & Male & Yes & No \\
\hline Clearly states what the service is & $36 \%$ & $39 \%$ & $40 \%$ & $24 \%$ & $32 \%$ & & $24 \%$ & $40 \%$ \\
\hline Name linked to the building & $34 \%$ & $31 \%$ & $32 \%$ & $29 \%$ & $33 \%$ & $17 \%$ & $38 \%$ & $28 \%$ \\
\hline $\begin{array}{l}\text { No reference to what/where } \\
\text { service is }\end{array}$ & $31 \%$ & $30 \%$ & $28 \%$ & & $34 \%$ & $33 \%$ & $38 \%$ & $32 \%$ \\
\hline
\end{tabular}

\section{P185 THE SEXUAL HEALTH OF TRANSGENDER WOMEN IN EAST LONDON}

Laurence Dufaur*, Jake Bayley. Bartshealth NHS Trust, London, UK

10.1136/sextrans-2016-052718.235

Background Previous studies into the sexual health of transgender women (TGW) report high rates of STI and HIV positivity. Aim To evaluate the sexual health of TGW attending routine GUM clinics in a London Trust.

Methods Retrospective case-note review of TGW attendances from May 2013 to November 2015. Clinical records and laboratory results assessed.

Results 52 attendances were made by 17 TGW with a median age of 31 years (IQR 27-36). 41.2\% were European, 52.9\% were White and $29.4 \%$ were Asian. All had sex with men however $23.5 \%$ also had sex with women. $17.6 \%$ report sex work in the last year but no unprotected anal intercourse (UAI) with clients. $64.7 \%$ report UAI with male partners in the preceding 3 months $(90.9 \%$ receptive). $64.7 \%$ had a history of any STI including $14.3 \%$ with Hepatitis B (naturally immune) and $6.7 \%$ with HIV. There were no diagnoses of Hepatitis C. The most common diagnosis made during the study period was Syphilis at $26.7 \%$ (of which 50\% early infection) followed by HPV $(23.5 \%)$, Chlamydia trachomatis $(18.8 \%)$, Neisseria gonorrhoea (18.8\%) and HSV (17.6\%). 35.3\% report drug or harmful alcohol use, $5.9 \%$ IVDU and $23.5 \%$ a history of physical or sexual assault.

Discussion Very high rates of UAI and STIs in TGW are comparable to those seen in previous studies. The prevalence of HIV infection is lower than expected from previous studies, perhaps due to variation in the cohort of TGW seen at our clinics. There remain significant challenges in identifying and providing tailored sexual health services to this at-risk population.

\section{P186 IMPROVING DIAGNOSIS OF GONORRHOEA: A SERVICE IMPROVEMENT PROJECT}

Emma Street ${ }^{*}$, Lindsay Short, Gavin Boyd. Calderdale and Huddersfield NHS Trust, Huddersfield, UK

10.1136/sextrans-2016-052718.236
Background With rising rates of gonorrhoea and increasing resistance, accurate diagnosis and appropriate use of antibiotics has become increasingly important. In response to this, we have focussed service improvement in our sexual health service (site $1=$ GUM clinic, site 2 = integrated clinic) over the past 5 years on gonorrhoea. Our main focus has been on the high level of NAAT positive, culture negative samples- was this related to false positive tests or failed culture or both. This prompted a review of how samples were handled and, in particular, the time period between sample taking for culture and arriving within the lab. We have refined procedures to improve uptake of culture testing, culture positivity and finally the addition of supplementary testing for all positive NAAT testing in 2015.

Aim To review gonorrhoea diagnosis over a 5 year period, exploring the issue of NAAT positive, culture negative samples.

Methods yearly audit of gonorrhoea diagnoses Results

\begin{tabular}{|c|c|c|c|c|c|c|}
\hline Year & 2011 & & 2013 & & 2014 & 2015 \\
\hline Number of cases & 195 & & 342 & & 342 & 189 \\
\hline Rate of GC/100000 & 46.1 (site1) & $\begin{array}{l}47.5 \\
\text { (site2) }\end{array}$ & $\begin{array}{l}51.4 \\
\text { (site1) }\end{array}$ & $\begin{array}{l}59.1 \\
\text { (site2) }\end{array}$ & $\begin{array}{ll}50.4 & 61.1 \\
\text { (site1) } & \text { (site2) }\end{array}$ & not available \\
\hline$\%$ cultures performed & 91 (site 1+ & & 60 (site & & 73 (site $1+2$ ) & 93 (site $1+2)$ \\
\hline$\%$ culture positive & 63 ( site $1+$ & & 52 (site & & 75 (site $1+2$ ) & 80 ( site $1+2$ ) \\
\hline
\end{tabular}

Discussion Gonorrhoea diagnoses have dramatically declined between 2014 and 2015 due to the introduction of supplementary testing to remove the issue of false positive results. We have improved the uptake of culture testing in the era of self-taken NAAT testing and improved culture positivity rate with simple changes in the processing of samples.

\section{P187 ESTIMATING COST SAVINGS BY INTRODUCING A REFLEX HEPATITIS B VIRUS SCREENING ALGORITHM IN A SEXUAL HEALTH SERVICE}

${ }^{1,2}$ Daniel Bradshaw*, ${ }^{3}$ David Muir, ${ }^{1}$ Michael Rayment. ${ }^{1}$ Chelsea and Westminster Hospital NHS Foundation Trust, London, UK; ${ }^{2}$ Brighton and Sussex University Hospitals NHS Trust, Brighton, UK; ${ }^{3}$ mperial College Healthcare NHS Trust, London, UK

\subsection{6/sextrans-2016-052718.237}

Background/introduction BASHH recommends that screening for HBV infection may be with $\mathrm{HBcAb}$, with reflex HBsAg testing in $\mathrm{HBcAb}$-positive patients. False negative $\mathrm{HBcAb}$ (eg in acute HBV infection or with low assay sensitivity) is rare. At the time our laboratory did not routinely perform reflex HBsAg testing, placing the onus on clinicians, many of whom therefore requested both tests simultaneously (with redundant sAg tests being performed in the presence of a negative $\mathrm{cAb}$ ). We wished to audit the extent of this practice and estimate cost savings by introducing reflex testing.

Aim(s)/objectives This was a retrospective case notes review of patients for whom HBcAb had been requested between 01/01/15 and $01 / 05 / 15$. The cost of performing HBsAg testing was estimated at $£ 3.60$ per test.

Methods There were two hundred patients with $\mathrm{HBcAb}$ results: 110 (55\%) male; median age 32 (IQR 26-39) years; 9 (4.5\%) HIV-infected. Twenty-two (11\%) tested HBcAb-positive of whom 5 (2.5\%) were HBsAg-positive, 16 (8.0\%) HBsAg- 